ARTICLE

Received 13 Nov 2014 | Accepted 27 Jan 2015 | Published 3 Mar 2015

DOI: $10.1038 /$ ncomms 7420

OPEN

\title{
Fabrication of three-dimensionally interconnected nanoparticle superlattices and their lithium-ion storage properties
}

Yucong Jiao,2, Dandan Han², Yi Ding1, Xianfeng Zhang ${ }^{1,2}$, Guannan Guo1, Jianhua Hu', Dong Yang ${ }^{1} \&$ Angang Dong ${ }^{2}$

Three-dimensional superlattices consisting of nanoparticles represent a new class of condensed materials with collective properties arising from coupling interactions between close-packed nanoparticles. Despite recent advances in self-assembly of nanoparticle superlattices, the constituent materials have been limited to those that are attainable as monodisperse nanoparticles. In addition, self-assembled nanoparticle superlattices are generally weakly coupled due to the surface-coating ligands. Here we report the fabrication of three-dimensionally interconnected nanoparticle superlattices with face-centered cubic symmetry without the presynthesis of the constituent nanoparticles. We show that mesoporous carbon frameworks derived from self-assembled supercrystals can be used as a robust matrix for the growth of nanoparticle superlattices with diverse compositions. The resulting interconnected nanoparticle superlattices embedded in a carbon matrix are particularly suitable for energy storage applications. We demonstrate this by incorporating tin oxide nanoparticle superlattices as anode materials for lithium-ion batteries, and the resulting electrochemical performance is attributable to their unique architectures.

\footnotetext{
${ }^{1}$ State Key Laboratory of Molecular Engineering of Polymers and Department of Macromolecular Science, Fudan University, Shanghai 200433, China.

${ }^{2}$ Department of Chemistry and Collaborative Innovation Center of Chemistry for Energy Materials, Fudan University, Shanghai 200433, China.

Correspondence and requests for materials should be addressed to D.Y. (email: yangdong@fudan.edu.cn) or to A.D. (email: agdong@fudan.edu.cn).
} 
T hree-dimensional (3D) superlattices consisting of nanoparticles (NPs) are emerging as a new and important class of nanostructured materials ${ }^{1}$, the properties of which can be rationally tuned by manipulating the size, shape and composition of the constituent NPs. In particular, interparticle interactions in NP superlattices can lead to collective properties, which are significantly different from the isolated NPs ${ }^{1-3}$. Existing methods of growing NP superlattices rely on the selfassembly of monodisperse colloidal NPs induced by solvent evaporation or antisolvent destabilization ${ }^{1}$. Recent progress on the self-assembly of NPs has been witnessed by the growth of a rich array of single- and multicomponent NP superlattices ${ }^{4-10}$, which have found wide applications in electronic and optoelectronic devices, catalysis and energy storage.

The self-assembly of colloidal NPs is a complicated process, involving various driving forces and interactions (for example, van der Waals, Coulombic, dipolar) ${ }^{1}$. As a consequence, successful assembly of long-range ordered NP superlattices requires exquisite control over many parameters, such as NP size distribution, surface-coating ligands and solvent evaporation kineticsand so on. In addition, self-assembled NP superlattices typically suffer from poor electrical conductivity due to the large interparticle spacing maintained by the capping ligands ${ }^{1,11}$. Therefore, post-surface treatment such as ligand exchange is required to enhance electronic coupling, which unfortunately can lead to severe structural defects such as cracks ${ }^{12,13}$. Another major bottleneck hampering prospects of NP superlattices is the limited choice of monodisperse NP building blocks, despite recent progress in colloidal synthesis ${ }^{1,14}$.

In this work we report an approach that can overcome the aforementioned limitations associated with self-assembly methods, enabling the growth of 3D interconnected, strongly coupled NP superlattices without the presynthesis of the constituent NPs. The resulting connected NP superlattices embedded in 3D continuous carbon frameworks represent a new class of superlattice materials, which are demonstrated to be remarkably promising for energy storage applications. $\mathrm{SnO}_{2} \mathrm{NP}$ superlattices are chosen as a model system to study Li-ion storage properties, which exhibit exceptional cycling stability and rate capability when used as Li-ion battery (LIB) anodes.

\section{Results}

Fabrication procedure. Figure 1a schematically illustrates the overall fabrication procedure. Briefly, starting with self-assembled $\mathrm{Fe}_{3} \mathrm{O}_{4} \mathrm{NP}$ supercrystals, we obtain 3D continuous, highly ordered mesoporous carbon frameworks on the carbonization of the surface-coating oleic acid (OA) ligands followed by the removal of $\mathrm{Fe}_{3} \mathrm{O}_{4}$ NPs. The subsequent impregnation of carbon frameworks with desired precursors leads to $3 \mathrm{D} \mathrm{NP}$ superlattices on hydrolysis and/or thermal annealing. The constituent NP composition can be modulated by selecting appropriate precursors, while the NP size can be tailored by controlling that of the original $\mathrm{Fe}_{3} \mathrm{O}_{4} \mathrm{NPs}$. The reason to select $\mathrm{Fe}_{3} \mathrm{O}_{4} \mathrm{NPs}$ as the starting material is that the scalable production of $\mathrm{Fe}_{3} \mathrm{O}_{4} \mathrm{NP}$ superlattices can be routinely achieved by self-assembly ${ }^{14,15}$. In addition, $\mathrm{Fe}_{3} \mathrm{O}_{4}$ is an inexpensive and thermally stable material, which can be readily removed by acid etching.

It is worth to mention that although the structure of our mesoporous carbon frameworks appears to be similar to that of previous mesoporous carbons prepared from hard template (that is, silica opals or mesoporous silica) ${ }^{16-22}$, we should emphasize that this is the first preparation of ordered mesoporous carbons from inorganic nanocrystals. There are several unique features associated with this preparation strategy, leading to mesoporous carbons with distinctive structural and textural properties. First, the surface-coating OA ligands, which are a necessary component for the growth and self-assembly of $\mathrm{Fe}_{3} \mathrm{O}_{4} \mathrm{NPs}$, concurrently serve as the carbon source, yielding mesoporous carbons having ultrathin pore walls $(\sim 2 \mathrm{~nm})$ and the same topological structure as the $\mathrm{Fe}_{3} \mathrm{O}_{4} \mathrm{NPs}$ supercrystal template. This is in sharp contrast with previous hard-templating approaches where additional carbon precursors (that is, polymers or prepolymers) have to be applied to fill the voids of the template and the resulting mesoporous carbons usually possess an inverse structure of the template $^{16-22}$. Furthermore, unlike previous approaches where silica opals or mesoporous silica only act as template ${ }^{16-22}$, the $\mathrm{Fe}_{3} \mathrm{O}_{4}$ NPs used here also serve as a graphitization catalyst (to be discussed below), allowing for partially graphitic frameworks at low temperatures $\left(500^{\circ} \mathrm{C}\right)$. More importantly, the unique structure of our carbon frameworks enables interconnected, strongly coupled NP superlattices, which could not be readily accessible by conventional self-assembly methods.

Mesoporous carbon frameworks. Monodisperse $\mathrm{Fe}_{3} \mathrm{O}_{4}$ NPs $(\sim 11 \mathrm{~nm})$ stabilized by $\mathrm{OA}$ are synthesized by a literature method ${ }^{14}$ (Supplementary Fig. 1a), and $\mathrm{Fe}_{3} \mathrm{O}_{4} \mathrm{NP}$ superlattices are grown by a conventional drying-mediated self-assembly process (Supplementary Fig. 1b) ${ }^{4}$. The content of the surfacecoating OA ligands determined from thermogravimetric analysis (TGA) is 15.9 wt\% (Supplementary Fig. 1c), corresponding to the area ligand coverage of $\sim 3.83 \mathrm{~nm}^{-2}$ (that is, $\sim 1455$ OA molecules per NP, Supplementary Note 1). The prior addition of squalane $(\sim 1 \mathrm{wt} \%)$ into the NP solution is found to favour the gram-scale growth of micrometer-sized NP supercrystals with well-developed facets ${ }^{15}$, although squalane is not critical for the subsequent formation of mesoporous carbon frameworks (Supplementary Fig. 1d). The as-assembled NP supercrystals are then heated at $500^{\circ} \mathrm{C}$ in an argon atmosphere, producing grey powders arising from the carbonization of OA (Supplementary Fig. 2a). The carbon species constitute $\sim 11 \mathrm{wt} \%$ of the carbonized NP supercrystals, determined from TGA (Supplementary Fig. 2b). Scanning electron microscopy (SEM) and highresolution SEM (HRSEM) establish that both the faceted morphology and the long-range NP ordering are well retained in carbonized $\mathrm{Fe}_{3} \mathrm{O}_{4} \mathrm{NP}$ supercrystals (Fig. 1b,c and Supplementary Fig. 2c-f). These results strongly suggest that cracks and other structural defects, which have been observed in many thermally treated NP superlattices reported previously ${ }^{23,24}$, are largely prevented in our experiments, probably due to the $3 \mathrm{D}$ crystal-like morphology as well as the long-range structural ordering of our $\mathrm{Fe}_{3} \mathrm{O}_{4} \mathrm{NP}$ supercrystals. The small-angle X-ray scattering (SAXS) pattern of carbonized $\mathrm{Fe}_{3} \mathrm{O}_{4} \mathrm{NP}$ supercrystals exhibits at least four scattering peaks, which can be assigned to the $111,220,311,420$ reflections of a well-crystallized facecentered cubic (fcc) structure (Fig. 1d, black curve), consistent with the highly ordered superlattice structure observed in HRSEM (Fig. 1c and Supplementary Fig. 2f). The unit cell parameter calculated from the SAXS data is $22.8 \mathrm{~nm}$.

$\mathrm{HNO}_{3}$ or $\mathrm{HCl}$ etching is employed to treat carbonized NP supercrystals. The complete removal of $\mathrm{Fe}_{3} \mathrm{O}_{4} \mathrm{NPs}$ yields black carbon powders after washing and drying (Supplementary Fig. 3a), which display a highly ordered porous structure as revealed by SEM (Supplementary Fig. 3b) and transmission electron microscopy (TEM, Fig. 1e,f and Supplementary Fig. 3c,d). SAXS (Fig. 1d, red curve) and SEM (Fig. 1e, inset) indicate that the porous carbon frameworks possess the same fcc structure and faceted morphology inherited from $\mathrm{Fe}_{3} \mathrm{O}_{4} \mathrm{NP}$ supercrystals, while the pore size $(\sim 10 \mathrm{~nm})$ is slightly smaller than the diameter of $\mathrm{Fe}_{3} \mathrm{O}_{4} \mathrm{NPs}$, probably caused by the framework shrinkage during etching and/or post drying 


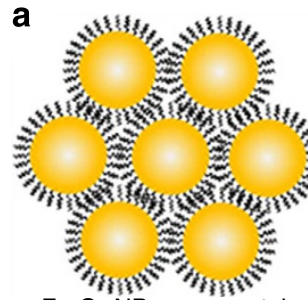

$\mathrm{Fe}_{3} \mathrm{O}_{4} \mathrm{NP}$ supercrystals

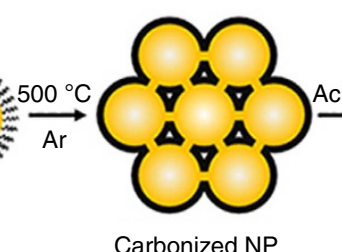

Supercrystals

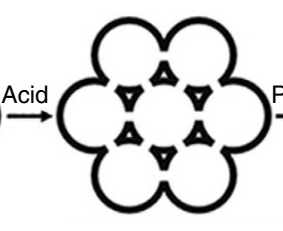

Mesoporous carbon frameworks

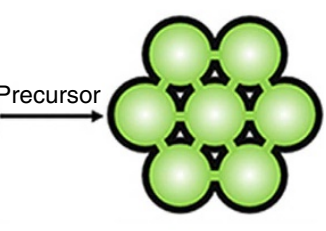

Interconnected NP superlattices
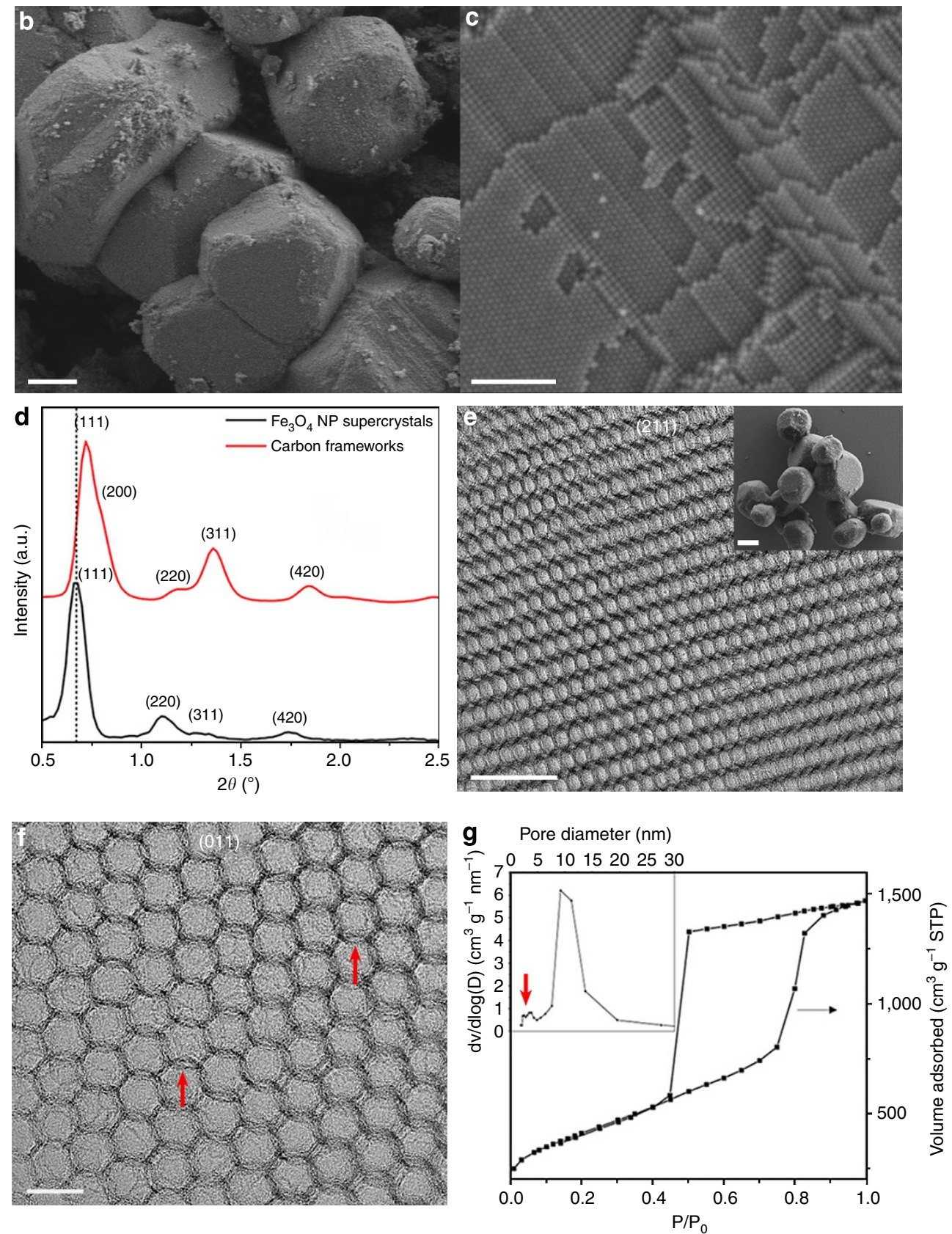

Figure 1 | Fabrication of three-dimensionally interconnected NP superlattices from mesoporous carbon frameworks. (a) Schematic illustration of the fabrication procedure (cross-sectional view). (b,c) SEM and HRSEM images of carbonized $\mathrm{Fe}_{3} \mathrm{O}_{4} \mathrm{NP}$ supercrystals, respectively. Scale bars, $1 \mu \mathrm{m}$ and $200 \mathrm{~nm}$, respectively. (d) SAXS patterns of carbonized $\mathrm{Fe}_{3} \mathrm{O}_{4} \mathrm{NP}$ supercrystals and mesoporous carbon frameworks, respectively. (e,f) TEM images of mesoporous carbon frameworks with different lattice projections. Scale bars, 50 and $20 \mathrm{~nm}$, respectively. The inset in (e) is a low-magnification SEM image of mesoporous carbon frameworks. Scale bar, $1 \mu \mathrm{m}$. The red arrows in (f) indicate the interconnected windows. (g) $\mathrm{N}_{2}$ adsorption-desorption isotherms and the corresponding pore size distribution (inset) of mesoporous carbon frameworks. The red arrow indicates the small pores corresponding to the interconnected windows observed in (f). 
processes. Interestingly, high-resolution TEM (HRTEM) and Raman spectroscopy suggest that the pore walls of carbon frameworks are partially graphitic (Supplementary Fig. 4), which is remarkable considering the low carbonization temperature $\left(500^{\circ} \mathrm{C}\right)$. We attribute the formation of partially graphitic frameworks at such a low temperature to the use of $\mathrm{Fe}_{3} \mathrm{O}_{4}$ $\mathrm{NPs}$, as transition metals such as $\mathrm{Fe}$ and $\mathrm{Co}$ and the corresponding metal oxides have been widely used as graphitization catalysts ${ }^{25-27}$. We surmise that the carbonaceous species arising from the thermal decomposition of OA molecules leads to the partial reduction of $\mathrm{Fe}_{3} \mathrm{O}_{4}$ NPs (most likely surface atoms) into metallic iron, which concurrently promotes the graphitization of the surrounding carbonaceous layers into partially graphitic pore walls. Despite the thin wall thickness $(\sim 2 \mathrm{~nm}$, Supplementary Fig. $4 \mathrm{~b}$ ), the graphitization degree of carbon frameworks can be further increased by heat treatment at $1,200^{\circ} \mathrm{C}$ in argon, converting the pore walls into few-layer graphene while retaining structural ordering (Supplementary Fig. 4c). More interestingly, tilted experiments along the [011] zone axis in TEM clearly shows that the adjacent pores are connected through small windows with dimensions of $\sim 3 \mathrm{~nm}$ (Fig. 1f, indicated by red arrows), leading to a $3 \mathrm{D}$ continuous porous structure. Presumably, the formation of the interconnected windows is attributed to the slight sintering of neighbouring $\mathrm{Fe}_{3} \mathrm{O}_{4} \mathrm{NPs}$ occurring during the carbonization process.

The porous structure of carbon frameworks is further characterized by $\mathrm{N}_{2}$ adsorption-desorption isotherms, which show a type-IV curve with a sharp capillary condensation step occurring in the relative pressure $\left(\mathrm{P} / \mathrm{P}_{0}\right)$ range of $0.8-0.85$ (Fig. 1g), a typical feature of mesoporous materials ${ }^{28}$. The Brunauer-Emmett-Teller surface area and the pore volume are determined to be $\sim 1,500 \mathrm{~m}^{2} \mathrm{~g}^{-1}$ and $\sim 2.5 \mathrm{~cm}^{3} \mathrm{~g}^{-1}$, respectively. The pore size distribution curve determined by using the BarrettJoyner-Halenda model suggests a bimodal porous structure (Fig. 1g, inset). The large pores at $\sim 10 \mathrm{~nm}$ correspond to the removed $\mathrm{Fe}_{3} \mathrm{O}_{4} \mathrm{NPs}$, while the small pores in the range of $2-4 \mathrm{~nm}$ are ascribed to the interconnected windows observed in TEM.

Interconnected NP superlattices. The 3D continuous porosity and high surface area of carbon frameworks are expected to facilitate the diffusion of precursors, which is crucial for the subsequent growth of NP superlattices. As a proof-of-concept demonstration, tetraethoxysilane is chosen as the precursor for $\mathrm{SiO}_{2} \mathrm{NP}$ superlattices, which are obtained by repeated precursor infiltration followed by hydrolysis. Remarkably, detailed structural features such as facets and surface terraces originated from $\mathrm{Fe}_{3} \mathrm{O}_{4} \mathrm{NP}$ supercrystals are also observed in the product (Fig. 2a,b and Supplementary Fig. 5), indicating a high degree of 3D NP ordering. The flexibility offered by the approach and the robustness of carbon frameworks allow the ready tuning of NP compositions by simply selecting appropriate precursors. For example, pure carbon NP superlattices can be realized by impregnating carbon frameworks with an aqueous solution of sucrose followed by drying and calcination (Fig. 2c,d). Energydispersive X-ray spectroscopy (EDS) and elemental mapping confirm that the resulting superlattices are composed of carbon NPs (Supplementary Fig. 6). To our knowledge, this is the first preparation of $3 \mathrm{D}$ carbon $\mathrm{NP}$ superlattices, as monodisperse, ligand-capped carbon NPs are not yet accessible for selfassembly ${ }^{29}$.

To further illustrate the generality of the approach, we fabricate superlattices of crystalline metal oxide NPs such as $\mathrm{SnO}_{2}$ by using $\mathrm{SnCl}_{2}$ as the precursor. Thermal annealing at $350^{\circ} \mathrm{C}$ in argon is carried out to crystallize the embedded NPs. TEM suggests that the crystallization process does not disrupt the ordered structure of $\mathrm{SnO}_{2}$ NP superlattices (Fig. 3a and Supplementary Fig. 7), while SAXS confirms the fcc geometry with a lattice constant of $21.2 \mathrm{~nm}$ (Fig. 3b). Powder X-ray diffraction (XRD, Fig. 3c) and HRTEM (Fig. 3d) establish that the constituent $\mathrm{SnO}_{2}$ NPs possess a high crystallinity with a tetragonal crystal structure. In addition to $\mathrm{SnO}_{2}$, other types of metal oxide nanocrystal superlattices can be prepared in a similar way using metal alkoxides or anhydrous inorganic salts as precursors. For instance, 3D superlattices consisting of anatase $\mathrm{TiO}_{2}$ nanocrystals are formed by impregnating carbon frameworks with titanium isopropoxide (TIP) followed by hydrolysis and thermal annealing (Supplementary Fig. 8). Moreover, NP superlattices of multicomponent phases, such as mixed oxides and metal phosphates, are also accessible by using a pre-mixed precursor (Supplementary Fig. 9). Apparently, the NP composition can be tailored by changing the ratio of the two precursors. For example, $\mathrm{Ti}_{0.3} \mathrm{Sn}_{0.7} \mathrm{O}_{2} \mathrm{NP}$ superlattices are obtained when a mixture of $\mathrm{SnCl}_{2}$ and TIP is employed as the precursor, and the uniform distribution of $\mathrm{Ti}$ and $\mathrm{Sn}$ as revealed by elemental mapping indicates that the homogenous filling of $\mathrm{Ti}_{0.3} \mathrm{Sn}_{0.7} \mathrm{O}_{2}$ NPs within the carbon framework (Supplementary Fig. 9c-e). The ability to fabricate such multiphase NP superlattices is particularly important, as they are generally hard to be prepared by self-assembly due to the challenge to obtain the corresponding monodisperse NPs.

Li-ion storage properties. One unique structural feature of NP superlattices produced by our approach is that the neighbouring NPs are connected with each other through interconnected windows within carbon frameworks, as shown in Fig. 3d where $\mathrm{SnO}_{2} \mathrm{NP}$ connections can be clearly observed (indicated by red arrows). Such connection configuration could substantially enhance electronic coupling between close-packed $\mathrm{NPs}^{30,31}$, which is highly desirable for applications requiring high electrical conductivity. In comparison to self-assembled NP superlattices, another important feature of our NP superlattices is that the constituent NPs are embedded in a continuous and partially graphitic carbon matrix. Such carbon coating could further facilitate electron transport and accommodate volume variations of NPs, which is particularly beneficial for energy storage devices ${ }^{32-34}$. To demonstrate this, $\mathrm{SnO}_{2} \mathrm{NP}$ superlattices are chosen as anode materials for LIBs, which exhibit excellent electrochemical performance in terms of cycling stability and rate capability. The reason to select $\mathrm{SnO}_{2} \mathrm{NP}$ superlattices is that $\mathrm{SnO}_{2}$ has attracted increasingly greater attention for $\mathrm{Li}$-ion storage due to its high theoretical capacity $\left(780 \mathrm{mAh}^{-1}\right)$ and environmental benignity ${ }^{35-44}$.

To investigate their electrochemical performance, LIB anodes based on $\mathrm{SnO}_{2} \mathrm{NP}$ superlattices are cycled on the basis of the half-cell configuration. The carbon content of $\mathrm{SnO}_{2} \mathrm{NP}$ superlattices is $\sim 28 \mathrm{wt} \%$, determined from TGA (Supplementary Fig. 10a). Electron microscopies indicate that $\mathrm{SnO}_{2} \mathrm{NP}$ superlattices are uniformly distributed in the electrode with wellretained structural ordering before cycling (Supplementary Fig. 10b-d). Fig. 4a shows the cyclic voltammograms of $\mathrm{SnO}_{2}$ $\mathrm{NP}$ superlattices in the potential range from 3.0 to $0.005 \mathrm{~V}$ (versus $\mathrm{Li} / \mathrm{Li}^{+}$) at a scan rate of $0.5 \mathrm{mV} \mathrm{s}^{-1}$. The irreversible peak at $0.75 \mathrm{~V}$ in the first lithiation process is attributed to the reduction of $\mathrm{SnO}_{2}$ into $\mathrm{Sn}$ as well as the formation of a solid electrolyte interphase $(\mathrm{SEI})^{39}$. The two peaks at 0.7 and $1.3 \mathrm{~V}$ in the first delithiation process correspond to the de-alloying of $\mathrm{Li}_{x} \mathrm{Sn}$ and the partially reversible reaction of $\mathrm{SnO}_{2}$ with $\mathrm{Li}^{+}$, respectively, consistent with previous results ${ }^{37,38}$. The cycling stability of $\mathrm{SnO}_{2}$ NP superlattices is studied by galvanostatic charge/discharge. The first discharge process leads to a capacity of $1,570 \mathrm{mAhg}^{-1}$ at a relatively high current density of $600 \mathrm{mAg}^{-1}$, whereas the 


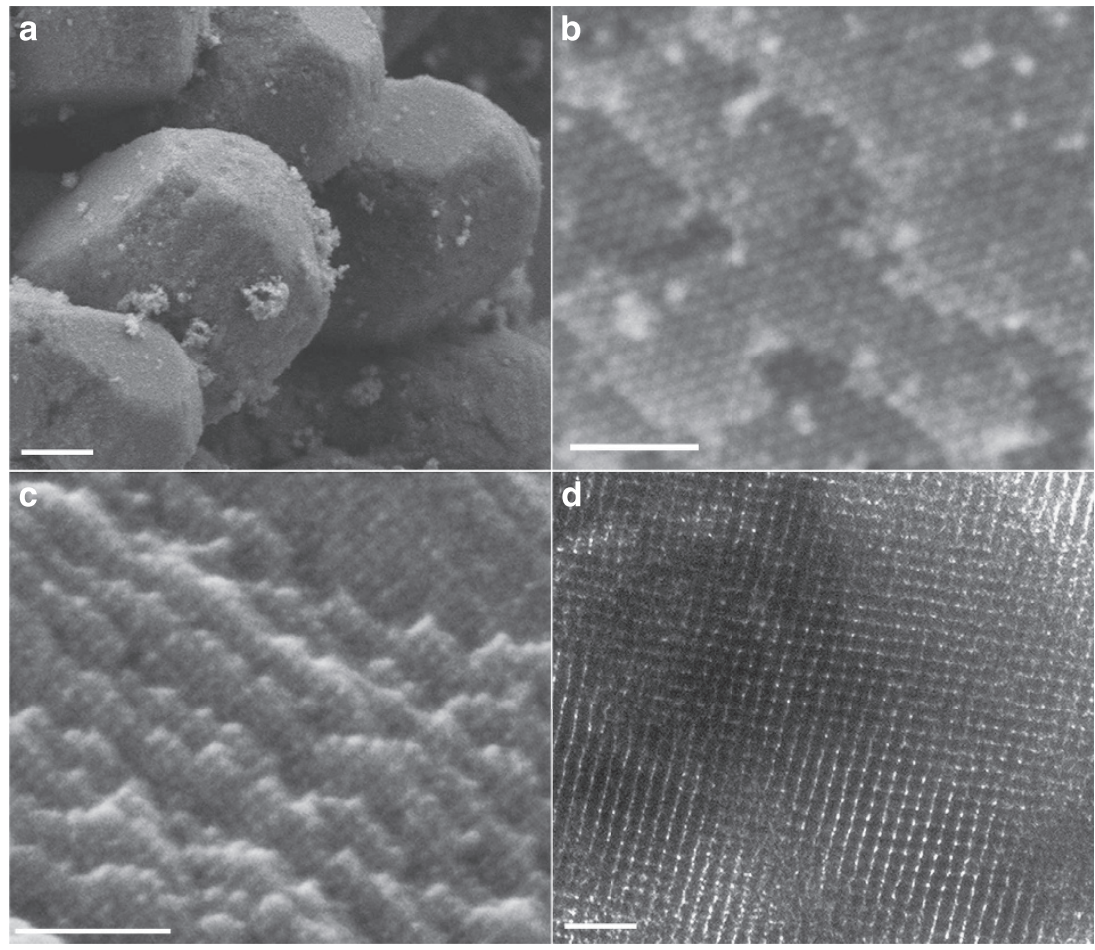

Figure 2 | Representative electron microscopy images of various NP superlattices. (a) SEM image of $\mathrm{SiO}_{2} \mathrm{NP}$ superlattices, showing the faceted morphology. Scale bar, $500 \mathrm{~nm}$. (b) HRSEM image of $\mathrm{SiO}_{2} \mathrm{NP}$ superlattices, showing the surface terraces and long-range NP ordering. Scale bar, $100 \mathrm{~nm}$. (c,d) SEM and TEM images of 3D carbon NP superlattices, respectively. Scale bars, 100 and $50 \mathrm{~nm}$, respectively.

a

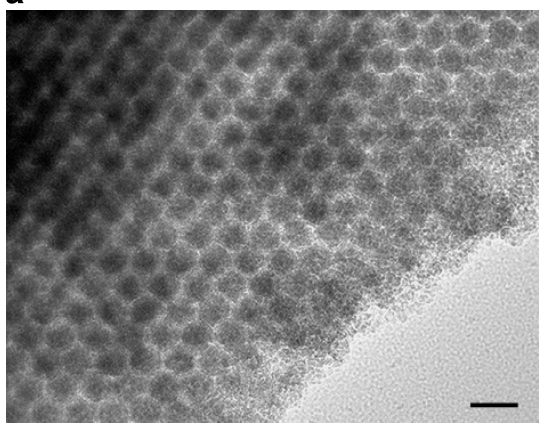

C

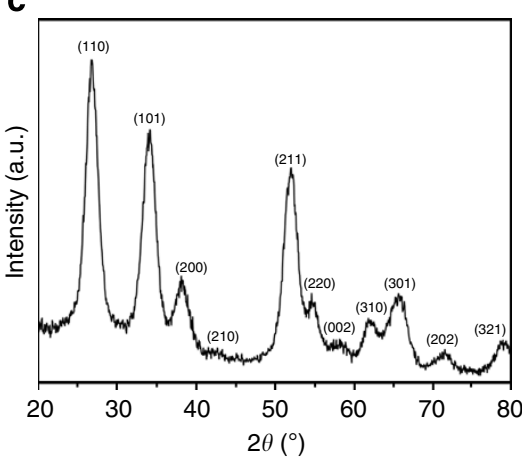

b

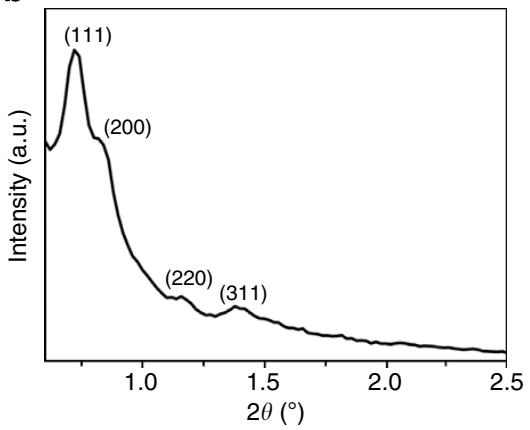

d

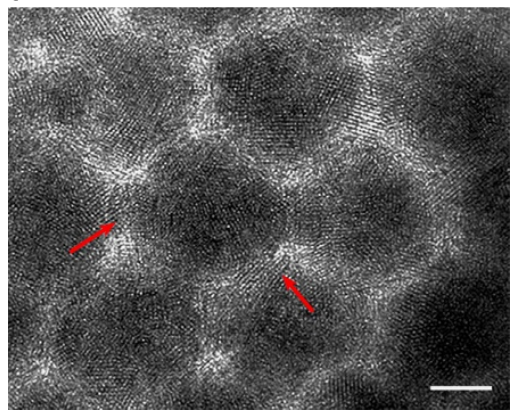

Figure 3 | Structural characterization of $\mathrm{SnO}_{\mathbf{2}}$ NP superlattices. (a) TEM image of $\mathrm{SnO}_{2}$ NP superlattices. Scale bar, $20 \mathrm{~nm}$. (b,c) SAXS pattern and XRD pattern of $\mathrm{SnO}_{2} \mathrm{NP}$ superlattices, respectively. (d) HRTEM image of $\mathrm{SnO}_{2} \mathrm{NP}$ superlattices, showing the high crystallinity of the embedded $\mathrm{SnO} \mathrm{NP}_{2} \mathrm{NP}$. Scale bar, $5 \mathrm{~nm}$. The red arrows indicate NP interconnections.

subsequent charge process delivers a capacity of $676 \mathrm{mAhg}^{-1}$ (Supplementary Fig. 11). This irreversibility is probably caused by the SEI formation as well as the lithium insertion into carbon frameworks ${ }^{36}$. Despite the first-cycle capacity loss commonly observed for $\mathrm{SnO}_{2}$ anodes ${ }^{36,37}$, our $\mathrm{SnO}_{2}$ NP superlattices exhibit excellent cycling stability, as manifested by the retention of a 

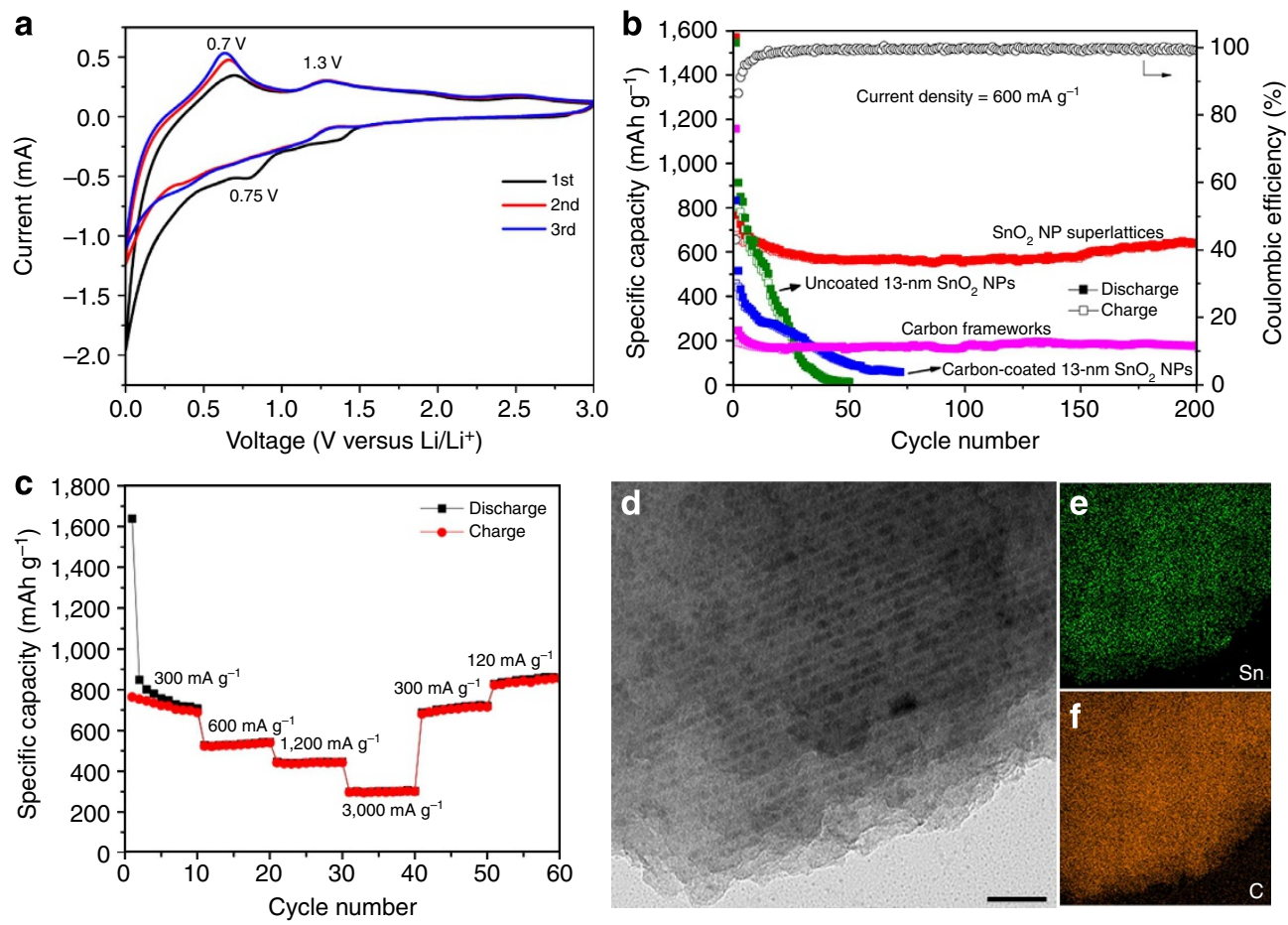

Figure 4 | Electrochemical characterization of $\mathbf{S n O}_{\mathbf{2}} \mathbf{N P}$ superlattices. (a) Representative cyclic voltammograms at a scan rate of $0.5 \mathrm{mV} \mathrm{s}^{-1}$. (b) $\mathrm{Cycling}$ performance at a current density of $600 \mathrm{mAg}^{-1}$ and the corresponding Coulombic efficiency. The cycling performance of $13-\mathrm{nm} \mathrm{SnO} 2 \mathrm{NPs}$ with and without carbon coating as well as carbon frameworks tested under the same conditions are also included for comparison. (c) Rate-capability test at current densities ranging from 120 to $3,000 \mathrm{mAg}^{-1}$. (d) TEM image and $(\mathbf{e}, \mathbf{f})$ the corresponding EDS elemental mapping of $\mathrm{SnO}_{2} \mathrm{NP}$ superlattices after 200 cycles, showing the preservation of ordered structure without NP aggregation. Scale bar, $50 \mathrm{~nm}$.

specific capacity of $640 \mathrm{mAh}^{-1}$ after 200 cycles and a stabilized Coulombic efficiency over $98 \%$ from the 10 th cycle (Fig. 4 b). LIB anodes based on mesoporous carbon frameworks only are also tested under the same conditions, which exhibit a stable capacity at $185 \mathrm{mAh} \mathrm{g}^{-1}$ after 200 cycles (Fig. 4b, pink curve), indicating that the high capacity of our $\mathrm{SnO}_{2} \mathrm{NP}$ superlattices is primarily attributed to the embedded $\mathrm{SnO}_{2}$ NPs. To better evaluate the charge/discharge performance of our $\mathrm{SnO}_{2} \mathrm{NP}$ superlattices, we synthesize colloidal $\mathrm{SnO}_{2} \mathrm{NPs}$ with a similar diameter $(\sim 13 \mathrm{~nm}$, Supplementary Fig. 12 $)^{45}$, which are cycled under the same conditions in control experiments. As expected, the uncoated $\mathrm{SnO}_{2}$ NPs exhibit fast capacity decay within 50 cycles (Fig. 4 b, olive curve), presumably caused by the pulverization and/or aggregation of $\mathrm{SnO}_{2} \mathrm{NPs}$ in the absence of carbon protection. In comparison, the carbon-coated $\mathrm{SnO}_{2} \mathrm{NPs}$ (carbon content: $\sim 26$ wt\%) exhibit better cycling stability, but their capacity rapidly fades below $100 \mathrm{mAh} \mathrm{g}^{-1}$ after 50 cycles (Fig. 4b, blue curve). The cycling performance of our $\mathrm{SnO}_{2} \mathrm{NP}$ superlattices is further evaluated at different current densities. As shown in Fig. 4c, the electrode is able to deliver a capacity of $300 \mathrm{mAhg}^{-1}$ even at a current density of $3,000 \mathrm{mAg}^{-1}$, and a capacity above $850 \mathrm{mAhg}^{-1}$ is recovered when the current density is switched back to $120 \mathrm{mAg}^{-1}$. Such cycling stability and rate capability outperform those of most $\mathrm{SnO}_{2} \mathrm{NP}$ anodes reported previously $^{38-44}$.

\section{Discussion}

The superior battery performance of $\mathrm{SnO}_{2} \mathrm{NP}$ superlattices is attributed to their unique structural characteristics. First, our NP superlattices exist as micrometer-sized secondary particles inherited from the original $\mathrm{Fe}_{3} \mathrm{O}_{4} \mathrm{NP}$ supercrystals, which are believed to be the ideal architectures for LIBs due to the reduced interfacial areas between active materials and electrolyte ${ }^{46,47}$. Second, and perhaps most importantly, the 3D graphitic carbon frameworks combined with interconnected NPs provide a continuous electron pathway, facilitating electronic connectivity within the components of the electrode. Moreover, the inherent flexibility of carbon frameworks can buffer large volume expansions of the embedded $\mathrm{SnO}_{2}$ NPs during cycling ${ }^{35,36}$, reducing the strain of the entire electrode.

To investigate the structural evolution of our $\mathrm{SnO}_{2} \mathrm{NP}$ superlattices during cycling, ex situ XRD, SEM, TEM, and EDS elemental mapping studies are performed after 200 cycles. In accordance with previous results for $\mathrm{SnO}_{2}$-based anodes ${ }^{35,43}$, XRD confirms the conversion of $\mathrm{SnO}_{2}$ NPs into $\mathrm{Sn}$ NPs after cycling (Supplementary Fig. 13a), while the broad diffraction peaks imply that the resulting Sn NPs are small in size without agglomeration. SEM reveals that the secondary particle morphology is largely preserved after cycling (Supplementary Fig. 13b,c), while TEM confirms that the Sn-based NPs are homogeneously confined within carbon frameworks with good structural ordering without aggregation (Fig. $4 \mathrm{~d}-\mathrm{f}$ and Supplementary Fig. 13d). These results strongly suggest that our carbon frameworks effectively suppress the agglomeration and pulverization of Sn-based NPs, which could explain the superior cycling stability of our $\mathrm{SnO}_{2} \mathrm{NP}$ superlattices. It should also be noted that the LIB performance could be further improved by enhancing the electrical conductivity of carbon frameworks before precursor infiltration, which can be realized by increasing the graphitization degree via heat treatment as mentioned above.

In summary, we have developed a new approach to fabricate three-dimensionally interconnected, strongly coupled NP superlattices with fcc packing symmetry. The generality of the approach is illustrated by the growth of NP superlattices with 
various compositions including oxides, carbon, mixed oxides, and phosphates. We also demonstrate the successful use of $\mathrm{SnO}_{2} \mathrm{NP}$ superlattices as anode materials for LIBs, and the excellent cycling stability and rate capability are attributed to their unique architectures (that is, 3D continuous and graphitic carbon coating, NP interconnections, and supercrystal morphology). Given the fact that the fabrication procedure does not require the presynthesis of monodisperse NP building blocks, we anticipate that in the future a wider range of materials can be prepared as interconnected NP superlattices, which can find various applications in electronics, catalysis, and energy storage.

\section{Methods}

Materials. Oleic acid (OA, 90\%), squalane, and 1-octadecene (ODE, 90\%) were purchased from Aldrich. Sodium oleate was obtained from TCI. Iron chloride hexahydrate $\left(\mathrm{FeCl}_{3} \cdot 6 \mathrm{H}_{2} \mathrm{O}\right)$, titanium isopropoxide (TIP), anhydrous tin chloride $\left(\mathrm{SnCl}_{2}\right)$, anhydrous tin tetrachloride $\left(\mathrm{SnCl}_{4}\right)$ tetraethoxysilane (TEOS), anhydrous zirconium tetrachloride $\left(\mathrm{ZrCl}_{4}\right)$, Cetyl trimethyl ammonium bromide (CTAB), and triethyl phosphate were purchased from Aladdin. All chemicals were used as received without further purification.

Synthesis and self-assembly of $\mathbf{F e}_{3} \mathbf{O}_{4}$ NPs. Monodisperse, OA-stabilized $\mathrm{Fe}_{3} \mathrm{O}_{4}$ NPs with a diameter of $\sim 11 \mathrm{~nm}$ were synthesized according to the literature method $^{14}$. In a typical synthesis, $72 \mathrm{~g}$ of iron oleate and $11.4 \mathrm{~g}$ of OA were dissolved in $400 \mathrm{~g}$ of ODE in a three-neck flask, and the resulting solution was heated at $320^{\circ} \mathrm{C}$ under a $\mathrm{N}_{2}$ atmosphere for $1 \mathrm{~h}$. After cooling down to room temperature, ethanol and isopropanol were added to precipitate $\mathrm{Fe}_{3} \mathrm{O}_{4} \mathrm{NPs}$, and the precipitates were dispersed in hexane to form a stable colloidal solution with a concentration of $\sim 10 \mathrm{mg} \mathrm{ml}^{-1}$. To self-assemble $\mathrm{Fe}_{3} \mathrm{O}_{4} \mathrm{NP}$ supercrystals, squalane ( $\sim 1 \mathrm{wt} \%$ ) was added to the NP solution and the solvent was then allowed to evaporate under ambient conditions. The complete solvent evaporation yielded faceted $\mathrm{Fe}_{3} \mathrm{O}_{4} \mathrm{NP}$ supercrystals with dimensions on the micrometer scale.

Fabrication of three-dimensionally interconnected NP superlattices. The asassembled $\mathrm{Fe}_{3} \mathrm{O}_{4} \mathrm{NP}$ supercrystals were heated in a quartz tube furnace at $500^{\circ} \mathrm{C}$ for $2 \mathrm{~h}$ in argon, converting the surface-coating ligands into carbon. The carbo-

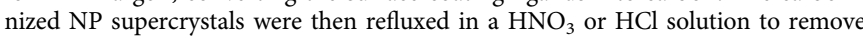
the embedded $\mathrm{Fe}_{3} \mathrm{O}_{4}$ NPs. The resulting mesoporous carbon frameworks retrieved by centrifugation were washed with deionized water, yielding black powders after drying. To grow interconnected NP superlattices, the dried porous carbon frameworks were infiltrated with desired precursors via wet impregnation followed by hydrolysis and/or thermal annealing.

Preparation of $\mathrm{SiO}_{2}$ NP superlattices. $5 \mathrm{mg}$ of dried mesoporous carbon powder was dissolved in $1 \mathrm{ml}$ of TEOS. After stirring for $6 \mathrm{~h}$, the precipitated powder collected by centrifugation was washed with ethanol to remove the excess TEOS. The hydrolysis of TEOS was induced by the addition of ammonia hydroxide, leading to the conversion of TEOS into $\mathrm{SiO}_{2}$. This impregnation/washing cycle was repeated twice in order to completely fill carbon frameworks with $\mathrm{SiO}_{2} \mathrm{NPs}$.

Preparation of carbon NP superlattices. 3D carbon NP superlattices were syn thesized using sucrose as the precursor. Briefly, $0.5 \mathrm{~g}$ of sucrose and $0.1 \mathrm{~g}$ of concentrated $\mathrm{H}_{2} \mathrm{SO}_{4}$ were first dissolved in $1 \mathrm{ml}$ of $\mathrm{H}_{2} \mathrm{O}$ to form a mixture, into which $5 \mathrm{mg}$ of dried mesoporous carbon powder was added with constant stirring. After $2 \mathrm{~h}$, the precipitated powder collected by centrifugation was washed with $\mathrm{H}_{2} \mathrm{O}$ to remove the excess sucrose and $\mathrm{H}_{2} \mathrm{SO}_{4}$. After that, the product was placed in an oven at $100^{\circ} \mathrm{C}$ for $2 \mathrm{~h}$ and then $160^{\circ} \mathrm{C}$ for another $2 \mathrm{~h}$. The black powder was then impregnated in a diluted sucrose solution $\left(0.05 \mathrm{~g}\right.$ of sucrose, $0.02 \mathrm{~g} \mathrm{of} \mathrm{H}_{2} \mathrm{SO}_{4}$, and $1 \mathrm{ml}$ of $\mathrm{H}_{2} \mathrm{O}$ ) for $2 \mathrm{~h}$. After washing and drying, the product was heated at $600{ }^{\circ} \mathrm{C}$ in argon for $2 \mathrm{~h}$ to carbonize sucrose, yielding $3 \mathrm{D}$ carbon NP superlattices.

Preparation of $\mathrm{SnO}_{2} \mathbf{N P}$ superlattices. $50 \mathrm{mg}$ of mesoporous carbon powder was mixed with a $\mathrm{SnCl}_{2}$ solution dissolved in ethanol $\left(0.1 \mathrm{~g} \mathrm{ml}^{-1}\right)$ and the resulting suspension was stirred for $2 \mathrm{~h}$. After that, the black powder was collected by centrifugation and the precipitated powder was washed with ethanol to remove the excess $\mathrm{SnCl}_{2}$. The precursor hydrolysis was initiated by the addition of ammonium hydroxide. This impregnation/washing cycle was repeated twice in order to completely load carbon frameworks with $\mathrm{SnO}_{2}$. Thermal annealing at $350{ }^{\circ} \mathrm{C}$ in argon for $1 \mathrm{~h}$ was carried out to crystallize the embedded $\mathrm{SnO}_{2} \mathrm{NPs}$.

Preparation of $\mathrm{TiO}_{2}$ NP superlattices. TIP was used as the precursor for the fabrication of $\mathrm{TiO}_{2} \mathrm{NP}$ superlattices. Briefly, $50 \mathrm{mg}$ of dried mesoporous carbon powder was mixed with a TIP solution dissolved in isopropanol $\left(0.5 \mathrm{~g} \mathrm{ml}^{-1}\right)$. After stirring for $6 \mathrm{~h}$, the black powder was collected by centrifugation and the precipitated powder was washed with isopropanol to remove the excess TIP. The subsequent hydrolysis of TIP was initiated by exposure to air, leading to the conversion of TIP into $\mathrm{TiO}_{2}$. This impregnation/washing cycle was repeated twice in order to completely fill carbon frameworks with $\mathrm{TiO}_{2}$. After drying, the product was heated at $350{ }^{\circ} \mathrm{C}$ in argon for $1 \mathrm{~h}$ to crystallize the embedded $\mathrm{TiO}_{2} \mathrm{NPs}$.

Preparation of $\mathbf{T i}_{\mathbf{x}} \mathbf{S n}_{\mathbf{1}} \mathbf{O}_{\mathbf{2}}$ NP superlattices. A homogeneous mixture of TIP and $\mathrm{SnCl}_{2}$ with different molar ratios dissolved in ethanol was used as the precursor for the growth of $\mathrm{Ti}_{x} \mathrm{Sn}_{1-x} \mathrm{O}_{2} \mathrm{NP}$ superlattices. In a typical synthesis of $\mathrm{Ti}_{0.3} \mathrm{Sn}_{0.7} \mathrm{O}_{2}$ NP superlattices, $0.4 \mathrm{mmol}$ of TIP and $0.6 \mathrm{mmol}$ of $\mathrm{SnCl}_{2}$ were dissolved in $1 \mathrm{ml}$ of ethanol to form a homogeneous solution, into which $5 \mathrm{mg}$ of dried mesoporous carbon powder was added with stirring. After $24 \mathrm{~h}$, the product was centrifuged and washed with ethanol to remove the excess precursor. The precursor hydrolysis was initiated by the addition of ammonium hydroxide. This impregnation/washing cycle was repeated twice in order to completely load carbon frameworks with $\mathrm{Ti}_{0.3} \mathrm{Sn}_{0.7} \mathrm{O}_{2} \mathrm{NPs}$. After drying, the product was heated at $350^{\circ} \mathrm{C}$ in argon for $1 \mathrm{~h}$ to crystallize the embedded $\mathrm{Ti}_{0.3} \mathrm{Sn}_{0.7} \mathrm{O}_{2} \mathrm{NPs}$.

Preparation of zirconium phosphate NP superlattices. Zirconium phosphate (ZrP) NP superlattices were prepared by using a homogeneous mixture of $\mathrm{ZrCl}_{4}$ and triethyl phosphate as the precursor. Briefly, $0.45 \mathrm{mmol}$ of $\mathrm{ZrCl}_{4}$ and $0.45 \mathrm{mmol}$ of triethyl phosphate were mixed in $2 \mathrm{ml}$ of ethanol to form a homogeneous solution, into which $5 \mathrm{mg}$ of dried mesoporous carbon powder was added with vigorous stirring for $2 \mathrm{~h}$. After that, the product was centrifuged and washed with ethanol to remove the excess precursor. The precipitated powder was then put in an oven at $110^{\circ} \mathrm{C}$ for $2 \mathrm{~h}$ to induce the formation of $\mathrm{ZrP}$ NP superlattices.

Synthesis of 13-nm $\mathrm{SnO}_{2}$ NPs in control experiments. In control experiments colloidal $\mathrm{SnO}_{2} \mathrm{NPs}$ with a similar size as our $\mathrm{SnO}_{2} \mathrm{NP}$ superlattices were synthesized by a literature method ${ }^{45}$. In a typical synthesis, $10 \mathrm{ml}$ of cationic surfactant (CTAB) solution $\left(0.08 \mathrm{moll}^{-1}\right)$ was mixed with $10 \mathrm{ml}$ of $\mathrm{NH}_{3} \cdot \mathrm{H}_{2} \mathrm{O}(25 \%$ aqueous solution) to form a homogeneous solution, into which $4.65 \mathrm{~g}$ of $\mathrm{SnCl}_{4}$ was added under vigorous stirring. After $4 \mathrm{~h}$, the product was filtered and washed with distilled water for several times. The product was then heated at $400{ }^{\circ} \mathrm{C}$ in air for $2 \mathrm{~h}$ to increase the crystallinity, leading to $\mathrm{SnO}_{2} \mathrm{NPs}$ with a mean diameter of $13 \mathrm{~nm}$. Sucrose was used as the carbon source for the formation of carbon-coated $\mathrm{SnO}_{2}$ nanocomposite. In a typical preparation of $\mathrm{SnO}_{2} / \mathrm{C}$ nanocomposite with a carbon content of $\sim 26 \mathrm{wt} \%, 0.35 \mathrm{~g}$ of $\mathrm{SnO}_{2} \mathrm{NPs}$ was mixed with a sucrose solution $\left(0.4 \mathrm{~g}\right.$ in $\left.5 \mathrm{ml} \mathrm{H}_{2} \mathrm{O}\right)$, and the resulting mixture was pre-heated at $180^{\circ} \mathrm{C}$ in an oven for $3 \mathrm{~h}$. The dried powder was then heated in a quartz furnace at $500^{\circ} \mathrm{C}$ for $5 \mathrm{~h}$ in argon, resulting into carbon-coated $\mathrm{SnO}_{2}$ nanocomposite.

Instrumentation. TEM images, HRTEM images, scanning TEM images, elemental mapping, and EDS spectra were obtained using a Tecnai G2 20 TWIN microscope operated at $200 \mathrm{kV}$. SEM images and EDS spectra were recorded using a Zeiss Ultra55 microscope operated at 5 and $10 \mathrm{kV}$, respectively. XRD was carried out on a Bruker D4 X-ray diffractometer, while SAXS was performed on a Nanostar U smallangle X-ray scattering system using $\mathrm{Cu} \mathrm{K} \alpha$ radiation $(40 \mathrm{kV}, 35 \mathrm{~mA})$. Nitrogen adsorption-desorption isotherms were recorded on a Tristar 3000 instrument. Before measurements, the samples were degassed at $300^{\circ} \mathrm{C}$ for $5 \mathrm{~h}$. Raman spectra were collected at room temperature on an XploRA Raman system. TGA measurements were carried out on a Perkin-Elmer Pyris 1 thermogravimetric analyzer.

Electrochemical measurements. The battery performance was evaluated by galvanostatic cycling of 2025-type coin cells assembled in an argon-filled glove box, with $\mathrm{SnO}_{2} \mathrm{NP}$ superlattices as the working electrode and lithium foil as the counter and reference electrode. The electrolyte was a $1.0 \mathrm{M} \mathrm{LiPF}_{6}$ solution in a mixture of ethylene carbonate, dimethyl carbonate and ethyl methyl carbonate (1:1:1 in volume), and a polypropylene film (Celgard-2300) was used as the separator. The working electrodes were prepared by a slurry-coating procedure. The slurry consisted of $\mathrm{SnO}_{2} \mathrm{NP}$ superlattices, acetylene black (Super P) and polyvinylidene fluoride binder with a mass ratio of 7:2:1 dissolved in $N$-methyl-2-pyrrolidinone. This slurry was spread on a copper foil, which acted as a current collector. The electrodes were dried at $90^{\circ} \mathrm{C}$ for $4 \mathrm{~h}$ in air, and then at $90^{\circ} \mathrm{C}$ in vacuum for another $12 \mathrm{~h}$. Cyclic voltammetry was carried out on an electrochemical workstation (Autolab 204 N), while galvanostatic measurements were performed on a Neware cell test instrument, which was cycled between 0.005 and $3.00 \mathrm{~V}$ (versus Li/ $\mathrm{Li}^{+}$) at various current densities.

\section{References}

1. Talapin, D. V., Lee, J.-S., Kovalenko, M. V. \& Shevchenko, E. V. Prospects of colloidal nanocrystals for electronic and optoelectronic applications. Chem. Rev. 110, 389-458 (2010).

2. Panthani, M. G. \& Korgel, B. A. Nanocrystals for electronics. Annu. Rev. Chem. Biomol. Eng. 3, 287-311 (2012).

3. Pileni, M. P. Self-assembly of inorganic nanocrystals: fabrication and collective intrinsic properties. Acc. Chem. Res. 40, 685-693 (2007). 
4. Murray, C. B., Kagan, C. R. \& Bawendi, M. G. Synthesis and characterization of monodisperse nanocrystals and close-packed nanocrystal assemblies. Annu. Rev. Mater. Sci. 30, 545-610 (2000).

5. Shevchenko, E. V., Talapin, D. V., Kotov, N. A., O’Brien, S. \& Murray, C. B. Structural diversity in binary nanoparticle superlattices. Nature 439, 55-59 (2006).

6. Kalsin, A. M. et al. Electrostatic self-assembly of binary nanoparticle crystals with a diamond-like lattice. Science 312, 420-424 (2006).

7. Mueggenburg, K. E., Lin, X. M., Goldsmith, R. H. \& Jaeger, H. M. Elastic membranes of close-packed nanoparticle arrays. Nat. Mater. 6, 656-660 (2007).

8. Dong, A. G., Chen, J., Patrick, M. V., Kikkawa, J. M. \& Murray, C. B. Binary nanocrystal superlattice membranes self-assembled at the liquid-air interface. Nature 466, 474-477 (2010).

9. Wang, T. et al. Self-assembled colloidal superparticles from nanorods. Science 338, 358-363 (2012).

10. Singh, G. et al. Self-assembly of magnetite nanocubes into helical superstructures. Science 345, 1149-1153 (2014).

11. Dong, A. G., Jiao, Y. C. \& Milliron, D. J. Electronically coupled nanocrystal superlattice films by in situ ligand exchange at the liquid-air interface. ACS Nano 7, 10978-10984 (2013).

12. Talapin, D. V. \& Murray, C. B. PbSe nanocrystal solids for $\mathrm{n}$ - and $\mathrm{p}$-channel thin film field-effect transistors. Science 310, 86-89 (2005).

13. Luther, J. M. et al. Structural, optical and electrical properties of self-assembled films of PbSe nanocrystals treated with 1,2-ethanedithiol. ACS Nano 2, 271-280 (2008).

14. Park, J. et al. Ultra-large-scale syntheses of monodisperse nanocrystals. Nat. Mater. 3, 891-895 (2004).

15. Nagaoka, Y., Chen, O., Wang, Z. W. \& Cao, Y. C. Structural control of nanocrystal superlattices using organic guest molecules. J. Am. Chem. Soc. 134, 2868-2871 (2012).

16. Cychosz, K. A. et al. Characterization of the pore structure of threedimensionally ordered mesoporous carbons using high resolution gas sorption. Langmuir 28, 12647-12654 (2012).

17. Gierszal, K. P. \& Jaroniec, M. Carbons with extremely large volume of uniform mesopores synthesized by carbonization of phenolic resin film formed on colloidal silica template. J. Am. Chem. Soc. 128, 10026-10027 (2006).

18. Lee, H. I. et al. Spontaneous phase separation mediated synthesis of 3D mesoporous carbon with controllable cage and window size. Adv. Mater. 23, 2357-2361 (2011).

19. Vu, A. et al. Three-dimensionally ordered mesoporous (3DOm) carbon materials as electrodes for electrochemical double-layer capacitors with ionic liquid electrolytes. Chem. Mater. 25, 4137-4148 (2013).

20. Fang, B., Kim, M., Kim, J. H., Lim, S. \& Yu, J. Ordered multimodal porous carbon with hierarchical nanostructure for high Li storage capacity and good cycling performance. J. Mater. Chem. 20, 10253-10259 (2010).

21. Jun, S. et al. Synthesis of new, nanoporous carbon with hexagonally ordered mesostructure. J. Am. Chem. Soc. 122, 10712-10713 (2000).

22. Kim, T. W., Park, I. S. \& Ryoo, R. A synthetic route to ordered mesoporous carbon materials with graphitic pore walls. Angew. Chem. Int. Ed. 42, 4375-4379 (2003).

23. Chen, J. et al. Bistable magnetoresistance switching in exchange-coupled $\mathrm{CoFe}_{2} \mathrm{O}_{4}-\mathrm{Fe}_{3} \mathrm{O}_{4}$ binary nanocrystal superlattices by self-assembly and thermal annealing. ACS Nano 7, 1478-1486 (2013).

24. Law, M. et al. Structural, optical, and electrical properties of PbSe nanocrystal solids treated thermally or with simple amines. J. Am. Chem. Soc. 130, 5974-5985 (2008)

25. Zhang, L. S., Li, W., Cui, Z. M. \& Song, W. G. Synthesis of porous and graphitic carbon for electrochemical detection. J. Phys. Chem. C 113, 20594-20598 (2009).

26. Lee, K. T., Ji, X., Rault, M. \& Nazar, L. F. Simple synthesis of graphitic ordered mesoporous carbon materials by a solid-state method using metal phthalocyanines. Angew. Chem. Int. Ed. 48, 5661-5665 (2009).

27. Gao, W., Wan, Y., Dou, Y. \& Zhao, D. Synthesis of partially graphitic ordered mesoporous carbons with high surface areas. Adv. Energy Mater. 1, 115-123 (2011).

28. Wan, Y. \& Zhao, D. Y. On the controllable soft-templating approach to mesoporous silicates. Chem. Rev. 107, 2821-2860 (2007).

29. Sun, Y. P. et al. Quantum-sized carbon dots for bright and colorful photoluminescence. J. Am. Chem. Soc. 128, 7756-7757 (2006).

30. Zhang, H. et al. Surfactant ligand removal and rational fabrication of inorganically connected quantum dots. Nano Lett. 11, 5356-5361 (2011).

31. Boneschanscher, M. P. et al. Long-range orientation and atomic attachment of nanocrystals in 2D honeycomb superlattices. Science 344, 1377-1380 (2014).

32. Liu, N. et al. A pomegranate-inspired nanoscale design for large-volumechange lithium battery anodes. Nat. Nanotech. 9, 187-192 (2014).

33. Chockla, A. M. et al. Silicon nanowire fabric as a lithium ion battery electrode material. J. Am. Chem. Soc. 133, 20914-20921 (2011).
34. Jang, B. et al. Direct synthesis of self-assembled ferrite/carbon hybrid nanosheets for high performance lithium-ion battery anodes. J. Am. Chem. Soc. 134, 15010-15015 (2012).

35. Han, F., Li, W., Li, M. \& Lu, A. Fabrication of superior-performance $\mathrm{SnO}_{2} @ \mathrm{C}$ composites for lithium-ion anodes using tubular mesoporous carbon with thin carbon walls and high pore volume. J. Mater. Chem. 22, 9645-9651 (2012).

36. Jahel, A., Ghimbeu, C. M., Monconduit, L. \& Vix-Guterl, C. Confined ultrasmall $\mathrm{SnO}_{2}$ particles in micro/mesoporous carbon as an extremely long cycle-life anode material for Li-ion batteries. Adv. Energy Mater. 4, 1400025 (2014).

37. Liang, J. et al. Bowl-like $\mathrm{SnO}_{2} @$ carbon hollow particles as an advanced anode material for lithium-ion batteries. Angew. Chem. Int. Ed. 53, 1-6 (2014).

38. Lin, J. et al. Graphene nanoribbon and nanostructured $\mathrm{SnO}_{2}$ composite anodes for lithium Ion batteries. ACS Nano 7, 6001-6006 (2013).

39. Ding, S. J. et al. Formation of $\mathrm{SnO}_{2}$ hollow nanospheres inside mesoporous silica nanoreactors. J. Am. Chem. Soc. 133, 21-23 (2011).

40. Qiao, H. et al. Sonochemical synthesis of ordered $\mathrm{SnO}_{2} / \mathrm{CMK}-3$ nanocomposites and their lithium storage properties. ACS Appl. Mater. Interfaces 3, 3704-3708 (2011).

41. Lee, K. T., Lytle, J. C., Ergang, N. S., Oh, S. M. \& Stein, A. Synthesis and rate performance of monolithic macroporous carbon electrodes for lithium-ion secondary batteries. Adv. Funct. Mater. 15, 547-556 (2005).

42. Kim, C., Noh, M., Choi, M., Cho, J. \& Park, B. Critical size of a nano $\mathrm{SnO}_{2}$ electrode for Li-secondary battery. Chem. Mater. 17, 3297-3301 (2005).

43. Li, X. et al. Tin oxide with controlled morphology and crystallinity by atomic layer deposition onto graphene nanosheets for enhanced lithium storage. $A d v$. Funct. Mater. 22, 1647-1654 (2012).

44. Zhang, X., Jiang, B., Guo, J., Xie, Y. \& Tang, L. Large and stable reversible lithium-ion storages from mesoporous $\mathrm{SnO}_{2}$ nanosheets with ultralong lifespan over 1000 cycles. J. Power Sources 268, 365-371 (2014).

45. Wang, Y. D., Ma, C. L., Sun, X. D. \& Li, H. D. Preparation and characterization of $\mathrm{SnO}_{2}$ nanoparticles with a surfactant-mediated method. Nanotechnology 13, 565-569 (2002).

46. Lee, S. H. et al. Self-assembled $\mathrm{Fe}_{3} \mathrm{O}_{4}$ nanoparticle clusters as high-performance anodes for lithium ion batteries via geometric confinement. Nano Lett. 13, 4249-4256 (2013).

47. Oszajca, M. F., Bodnarchuk, M. I. \& Kovalenko, M. V. Precisely engineered colloidal nanoparticles and nanocrystals for Li-ion and Na-ion batteries: model systems or practical solutions? Chem. Mater. 26, 5422-5432 (2014).

\section{Acknowledgements}

D.Y. is grateful for financial support from Natural National Science Foundation of China (51103026, 51373035, and 51373040), the Shanghai Scientific and Technological Innovation Project (11JC1400600 and 124119a2400), and International Science and Technology Cooperation Program of China (2014DFE40130). A.D. acknowledges the financial support from Fudan University, the ' 1000 Youth Talents' Plan, Natural National Science Foundation of China (21373052), and National Basic Research Program of China (973 program: 2014CB845602).

\section{Author contributions}

Y.J. and A.D. conceived and designed the experiments. Y.J. and D.H. performed the experiments. Y.D., X.Z., G.G. and J.H. collected and analysed the XRD and TEM data. Y.J., D.Y. and A.D. wrote the paper. All authors discussed the results and commented on the manuscript.

\section{Additional information}

Supplementary Information accompanies this paper at http://www.nature.com/ naturecommunications

Competing financial interests: The authors declare no competing financial interests.

Reprints and permission information is available online at http://npg.nature.com/ reprintsandpermissions/

How to cite this article: Jiao, Y. et al. Fabrication of three-dimensionally interconnected nanoparticle superlattices and their lithium-ion storage properties. Nat. Commun. 6:6420 doi: $10.1038 /$ ncomms7420 (2015).

This work is licensed under a Creative Commons Attribution 4.0 International License. The images or other third party material in this article are included in the article's Creative Commons license, unless indicated otherwise in the credit line; if the material is not included under the Creative Commons license, users will need to obtain permission from the license holder to reproduce the material. To view a copy of this license, visit http://creativecommons.org/licenses/by/4.0/ 